\title{
I/Q IMBALANCE OF TWO-PATH LADDER FILTERS
}

\author{
Peter Kiss and Vladimir Prodanov \\ Department of Wireless Circuits Research, Agere Systems \\ 555 Union Blvd., Allentown, PA 18109, kpeter@agere.com
}

\begin{abstract}
The frequency-(in)dependent I/Q imbalance of analog filters is a significant contributor to the tight noise budget of high-performance wireless applications, such as 802.11a wireless LANs. This paper proposes a "frequency-dependent" statistical I/Q-imbalance analysis method for two-path filters, which can be configured for both low-IF and zero-IF architectures. A 7-pole complex band-pass ladder filter is analyzed, and it shows large image rejection ratio $(\mathrm{rms} I M R>43.7 \mathrm{~dB}$ for $3 \sigma$ ). The same filter, but reconfigured as a pair of real low-pass filters, achieved about 13-dB less IMR. These results suggest a low-IF architectural choice to combat the I/Q imbalance of two-path filters.
\end{abstract}

\section{MOTIVATION}

Single-sideband modulation has been a popular choice for modern communication systems. Low-if and zero-IF architectures offer the potential of highly-integrated transceivers [1]. However, the mismatch errors of the mixers, local oscillator, analog filters and data converters limit the achievable image rejection ratio $(I M R)$ [1]. Wide-band wireless applications, such as 802.11a wireless LANs [2], require antenna-referred noise figure as low as $6 \mathrm{~dB}$ and signal-tonoise-and-distortion ratio of about $26 \mathrm{~dB}$ for fading channels. Therefore, an overall $I M R$ of $35-40 \mathrm{~dB}$ should be met in the receiver (RX) chain over a large bandwidth of $17 \mathrm{MHz}$. A low-IF transmitter (TX) would need a total $I M R$ in excess of $40-45 \mathrm{~dB}$.

The I/Q-mismatch contribution of the analog filters might be significant to a tight noise budget. Although little can be done about IC-technology matching data besides careful layout, the issue can be handled by making an appropriate architectural choice. This paper shows that complex bandpass filters [3-8] (CBPF) and a pair of real low-pass filters $[9,10]$ (RLPF), used in low-IF and zero-IF transceivers [1], respectively, behave differently in the presence of circuitelement mismatch. A two-path 7th-order ladder filter prototype is presented in Sec. 2, and its I/Q imbalance is analyzed in Secs. 3 and 4. Discussions will precede the Conclusion.

\section{TWO-PATH LADDER PROTOTYPE}

As an illustrative example, a 7th-order CBPF has been designed. A 0.5-dB pass-band ripple all-pole Chebyshev trans-

Prepared for IEEE ISCAS-2004. The authors would like to acknowledge Mihai Banu and Jack Glas for useful discussions and support. fer function [9, p. 322] was chosen with a $17-\mathrm{MHz}$ bandwidth centered at a $10-\mathrm{MHz}$ intermediate frequency (IF). This gives adequate rejection in the channel-selection process for a low-IF 802.11a [2] wireless LAN. Complex ladder architecture has been used since ladder (as opposed to cascade-of-poles) filters are known to have very low sensitivity to component tolerances [10, p. 475]. Therefore, one expects good I/Q matching properties.

The linear model of the filter is shown in Fig. 1. The CBPF is built from two low-pass filters. Each $L C$ ladder prototype [9] was emulated by element replacement [10, Sec. 14] using active $G_{m}-C$ circuits [10, Sec. 16]. The lowpass filters are coupled by 14 transconductors $\left(g c_{i}^{k}\right)$. There is a termination transconductor $\left(g_{i}^{1,1}\right.$ and $\left.g_{i}^{7,7}\right)$ at each end of the filters. The nominal values for the circuit elements $\left(g_{i}^{k, l}, g c_{i}^{k}\right.$ and $\left.c_{i}^{k} ; i, k, l=1 \ldots 7\right)$ used in the complex filter are tabulated in Tab. 1. The values were obtained after node-voltage scaling and thermal-noise scaling - aiming for $>50-\mathrm{dB}$ dynamic range for $\mathrm{a} \pm 0.5-\mathrm{V}_{p p}$ differential input in a $2.5-\mathrm{V} 0.25-\mu \mathrm{m}$ CMOS technology.

Disconnecting the coupling between the two paths by setting $g c$ values to 0 , the CBPF becomes a pair of RLPF, which is ready to be used for a zero-IF architecture. Therefore, a fair I/Q imbalance comparison is possible between low-IF and zero-IF architectures, since the core of the filter stays the same but the transconductors $g c$ are activated or deactivated. Such a comparison will be carried out next.

\section{I/Q MISMATCH ANALYSIS}

A two-path filter (e.g., Fig. 1) can be considered as a twoinput two-output linear network with complex input $x_{c}(t) \doteq$ $x_{1}(t)+j x_{2}(t)$ and complex output $y_{c}(t) \doteq y_{1}(t)+j y_{2}(t)$. The output of a mismatched two-path filter is given by

$$
Y_{c}(\omega)=H_{c m}(\omega) X_{c}(\omega)+H_{d f}(\omega) X_{c}^{*}(-\omega) .
$$

Eq. (1) shows that the input complex signal $x_{c}(t)$ is processed in a parallel fashion by $h_{c m}(t)$ and $h_{d f}(t)[7,8]$. The common component of the two paths forms $H_{c m}(\omega)$ which gives the desired (direct) output $H_{c m}(\omega) \cdot X_{c}(\omega)$. However, when the gain and/or the phase of the two paths are not matched, then a nonzero $H_{d f}(\omega)$ contributes to a leakage (undesired or difference) output component $H_{d f}(\omega)$. $X_{c}^{*}(-\omega)[7,8]$. 


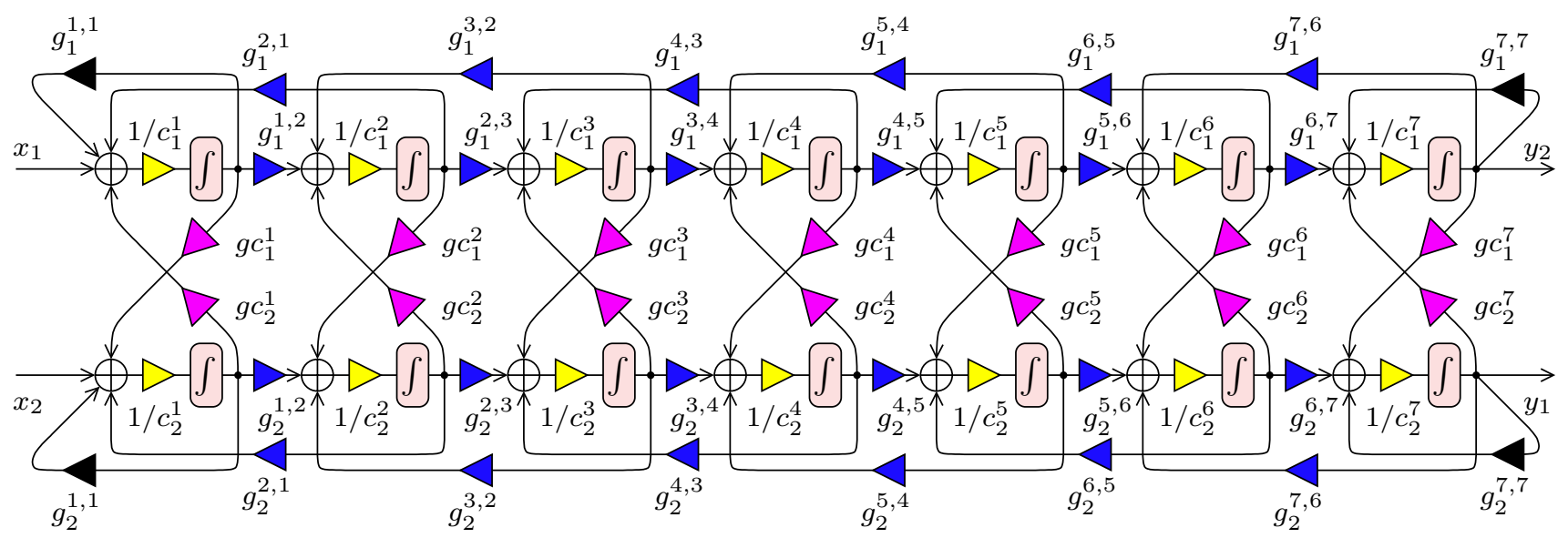

Figure 1: Linear model of a 7-pole complex ladder filter implemented by $G_{m}-C$ integrators. The signs of coefficients are given in Tab. 1 .

\begin{tabular}{|c|c|c|c|c|c|c|}
\hline \multicolumn{3}{|c|}{$\mathrm{g} 1=\mathrm{g} 2=$} & {$[\mathrm{uA} / \mathrm{v}]$} & \multicolumn{3}{|c|}{ (main transconductors) } \\
\hline$[-534.1$ & 191.6 & 0 & 0 & 0 & 0 & 0 \\
\hline-766.3 & 0 & 95.8 & 0 & 0 & 0 & 0 \\
\hline 0 & -191.6 & 0 & 67.7 & 0 & 0 & 0 \\
\hline 0 & 0 & -95.8 & 0 & 67.7 & 0 & 0 \\
\hline 0 & 0 & 0 & -67.7 & 0 & 67.7 & 0 \\
\hline 0 & 0 & 0 & 0 & -67.7 & 0 & 67.7 \\
\hline 0 & 0 & 0 & 0 & 0 & -67.7 & $-47.2]$ \\
\hline \multicolumn{3}{|c|}{$\mathrm{c} 1=\mathrm{c} 2=$} & {$[\mathrm{pF}]$} & \multicolumn{3}{|c|}{ (itegrating capacitors) } \\
\hline$[17.90$ & 6.67 & 3.40 & 2.52 & 2.40 & 2.36 & $1.58]$ \\
\hline \multicolumn{2}{|c|}{$\operatorname{gc} 1=-g c 2=$} & {$[\mathrm{uA} / \mathrm{v}]$} & \multicolumn{4}{|c|}{ (coupling transconductors) } \\
\hline [ 1124.5 & 419.2 & 213.3 & 158.2 & 2150.8 & 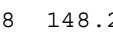 & 99.39] \\
\hline
\end{tabular}

Table 1: Nominal values for the complex ladder (Fig. 1).

For example, if a complex positive-frequency tone at $\omega_{0}$ undergoes an imperfect two-path filtering operation, then the complex output will contain, besides the desired component at $\omega_{0}$, a leakage component at $-\omega_{0}$ (Fig. 2). Similarly, a complex input tone at $-\omega_{0}$ will leak into $\omega_{0}$. Note that this distortion occurs independently from the leakage caused by the mixers, local oscillator and data converters. In practical situation all imperfections add [1].

Since the image rejection ratio, defined in $\mathrm{dB}$ as $\operatorname{IMR}(\omega)=20 \log _{10}\left|H_{c m}(\omega) / H_{d f}(\omega)\right|$, is a function of frequency, it is convenient to calculate for pass-band frequencies $(\omega \in B W)$ its rms average [8]

$I M R_{\mathrm{rms}}=10 \log _{10} \int_{\omega \in B W} \frac{1}{B W} \cdot\left|\frac{H_{c m}(\omega)}{H_{d f}(\omega)}\right|^{2} d \omega$

and its minimum $I M R_{\min }=\min \{I M R(\omega)\}_{\omega \in B W}$ values. In general, $I M R_{\mathrm{rms}}$ is a measure of the image (blocker) rejection in low-IF RX and of self distortion in zero-IF RX/TX. On the other hand, the value $I M R_{\min }$ is significant in meeting the mask specifications in low-IF TX. Also, the difference between $I M R_{\mathrm{rms}}$ and $I M R_{\mathrm{min}}$ is a measure of the frequency (in)dependency of $\operatorname{IMR}(\omega)$. When $\operatorname{IMR}(\omega)$ is significantly small and frequency dependent, then the necessary I/Q calibration, e.g., [11], becomes expensive.

\subsection{Complex BPF example}

The $I M R$ of a two-path filter is limited by matching between the circuit elements which implement the location of its poles and zeros, in this case, the transconductors $G_{m}$ and capacitors $C$. To simulate this effect all circuit elements (Fig. 1) were perturbed by a normally-distributed mismatch of $1 \%$ ( $3 \sigma$ value); the errors were assumed to be uncorrelated. One scenario of CBPF is shown in Fig. 3.

The simulations were performed using a black-box approach. In this method a perfect quadrature complex signal, i.e., $x_{c}(t)=A \cos \left(\omega_{0} t\right)+j A \sin \left(\omega_{0} t\right)$, was applied to the input of the filter. The spectrum of the resulting complex output $y_{c}(t)$ was measured at $\omega_{0}$ and $-\omega_{0}$, providing the values for $H_{c m}\left(\omega_{0}\right)$ and $H_{d f}\left(-\omega_{0}\right)$, respectively (Fig. 2). The experiment was performed for the range of frequencies of interest, i.e., -20 to $20 \mathrm{MHz}$.

Note that a more reliable estimate of the $I M R$ can be done at circuit level by Monte-Carlo runs, which extract the matching properties of all circuit elements (including biasing) from technology data. Here the results of system-level simulations are given, which provide a first-order approximation of the achievable $I M R$ and a good insight into twopath filters' behavior. However, when the authors applied this Matlab method to the filter reported in [12], the simulated $\operatorname{IMR}(\omega)$ results closely matched the measured ones.

\subsection{Real LPF example}

Next, a pair of RLPFs was simulated (Fig. 3). This two-path filter is exactly the same (including the mismatches) as the one used as a CBPF, except the coupling transconductors $g c$ were disabled. Therefore, the center frequency dropped
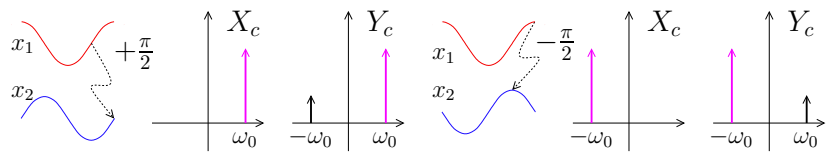

Figure 2: Imperfect filtering of a complex positive-frequency (left) and negative-frequency (right) input tone. 

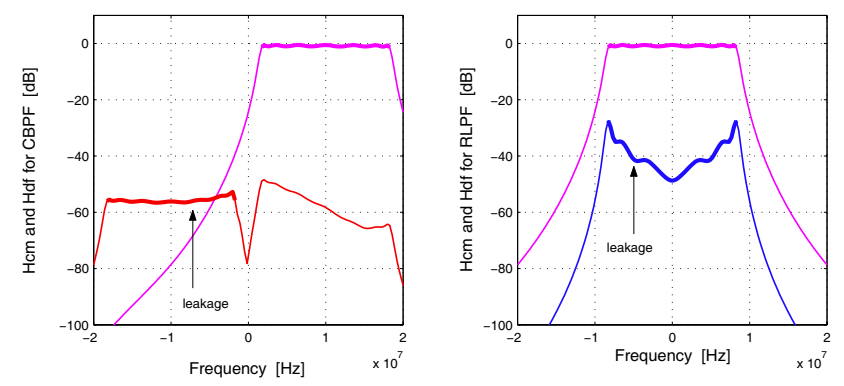

Figure 3: Desired and undesired responses for CBPF and RLPF.

from $10 \mathrm{MHz}$ to DC. Since the leakage is determined by the matching of two real low-pass filters, $I M R(\omega)$ becomes an even function of $\omega$, while the leakage is asymmetrical in $\omega$ for CBPF (Fig. 3). Note that the "image" is the signal itself in zero-IF architectures.

The $I M R_{\mathrm{rms}}$ and $I M R_{\mathrm{min}}$ values are $54.8 \mathrm{~dB}$ and $52.1 \mathrm{~dB}$ for CBPF, respectively, but they drop by about $20 \mathrm{~dB}$ to $35.6 \mathrm{~dB}$ and $26.1 \mathrm{~dB}$ for RLPF, respectively (Fig. 3). Taking into account the difference between $I M R_{\text {rms }}$ and $I M R_{\min }$, $\operatorname{IMR}(\omega)$ of the RLPF varies with about $7 \mathrm{~dB}$ more than $\operatorname{IMR}(\omega)$ of the CBPF. However, these are just partial results. In order to draw general conclusions, statistical analysis should be carried out - presented next.

\section{STATISTICAL ANALYSIS}

The experiment described in Sec. 3 was repeated for 2000 mismatch states (i.e., 2000 trials or 2000 realizations of the random mismatch process) and the results were processed statistically. First, $\operatorname{IMR}(\omega)$ is investigated as a function of frequency. The $\operatorname{IMR}(\omega)$ curves resulted from the 2000 trials are shown in Fig. 4 on top of each other forming a gray "background." The $I M R(\omega)$ curves were obtained using 112 complex test tones. Therefore, the $I M R(\omega)$ curves can be "sliced" into 112 frequency bins; each of them contains 2000 statistical $I M R$ values. The histogram of each frequency bin was calculated, thus the median (50\%), $1 \sigma$ $(65.87 \%)$ and $3 \sigma(99.74 \%)$ yield values were determined and plotted on Fig. 4. The distributions were not exactly Gaussian, so the 'median' was considered a more accurate average than the 'mean.'

\subsection{Qualitative evaluation}

It can be observed from Fig. 4 that the CBPF and RLPF statistical $I M R(\omega)$ curves look quite different. It can be concluded by inspection that the two types of filters cause fundamentally different I/Q imbalances in the presence of same circuit-element mismatch.

While RLPF have a statistically frequency-dependent $\operatorname{IMR}(\omega)$, CBPF exhibit a predominantly "flat" statistical $\operatorname{IMR}(\omega)$ in function of $\omega$. For CBPF, however, there is a deteriorative bend in the $\operatorname{IMR}(\omega)$ curve as the frequency approaches the pass band. Simulations show that this bend
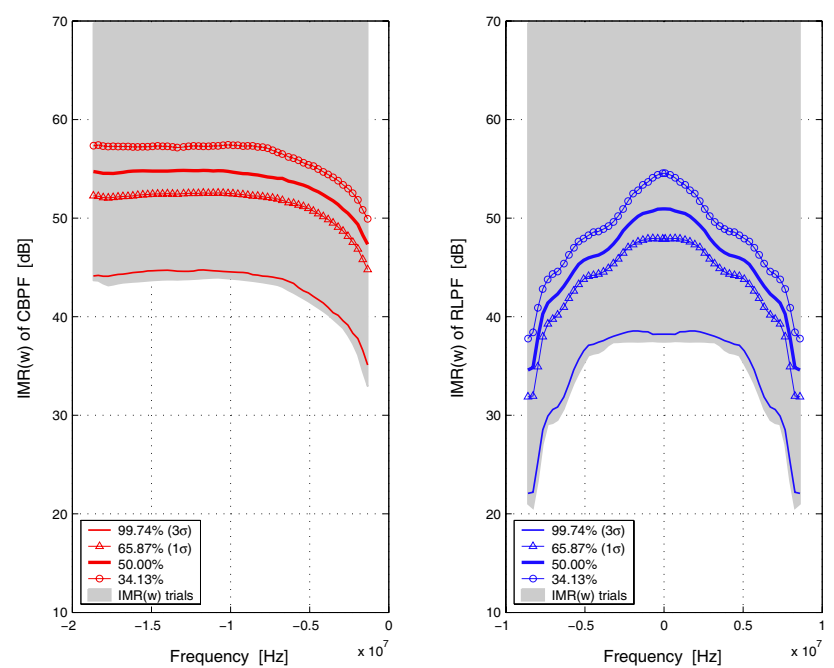

Figure 4: $\operatorname{IMR}(\omega)$ in function of $\omega$ for CBPF and RLPF.
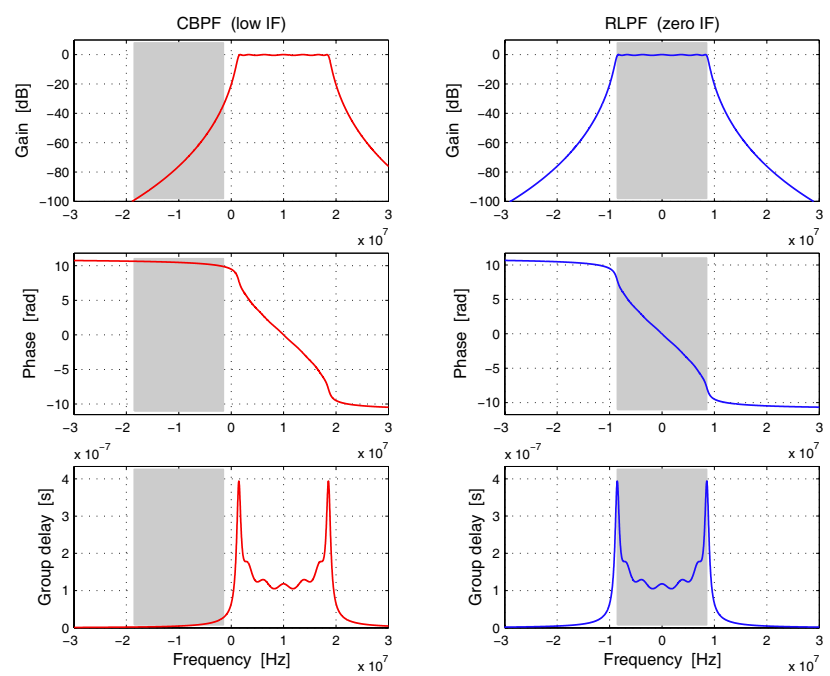

Figure 5: Frequency responses of CBPF and RLPF.

straightens out when the IF increases (e.g., from $10 \mathrm{MHz}$ to $20 \mathrm{MHz}$ ). However, using a higher IF is impractical for 802.11a applications due to blocker specifications [2].

Fig. 5 shows the frequency response of a CBPF and a pair of RLPFs. The gray area indicates the potential location of the image, which may interfere with the desired signal due to limited $I M R$. It is interesting to compare the shape of the group delay in the image band with the statistical $\operatorname{IMR}(\omega)$ curves presented in Fig. 4. Inspection indicates a high correlation between the two.

The bend in the CBPF's $I M R(\omega)$ curve (Fig. 4), therefore, is due to the small phase variations in the upper (close to DC) portion of the image band. It seems that the gain imbalances average out to a frequency-independent I/Q error.

Are the ripples of the RLPF's $I M R(\omega)$ curve (Fig. 4) caused by the high pass-band (= image-band) phase sensitivity? Additional analysis showed that both phase and gain 
imbalances contribute to $\operatorname{IMR}(\omega)$. More precisely, phase errors dominate the $I M R$ near the edges of the pass/image band, but gain errors are the dominant $I M R$ contributor around DC, i.e., at low frequencies. The "bumpy" behavior of the $\operatorname{IMR}(\omega)$ can be explained by the increased sensitivity of the complex transfer function in the vicinity of the poles.

\subsection{Quantitative comparison}

The $2000 \operatorname{IMR}(\omega)$ curves showed in Fig. 4 allow determining the yield of such filters. For CBPF $I M R_{\mathrm{rms}}$ and $I M R_{\min }$ for $3 \sigma$ certainty ( $99.74 \%$ yield) are $43.7 \mathrm{~dB}$ and $35.1 \mathrm{~dB}$, respectively (Fig. 6). These values are encouraging, since they may fit well into a low-IF 802.11a RX noise budget. In other words, by using such a CBPF the required I/Q calibration for the RX may be relaxed to a frequencyindependent calibration to correct the errors of the front end (i.e., mainly gain errors in the mixers, and phase errors in the local oscillators [1]).

In case of RLPF, the $I M R_{\mathrm{rms}}$ distribution is getting wider and it is shifted towards lower values (Fig. 6). The $I M R_{\text {rms }}$ and $I M R_{\min }$ for $3 \sigma$ certainty are $30.9 \mathrm{~dB}$ and $22.1 \mathrm{~dB}$, respectively. Unfortunately, the $I M R$ offered by the pair of RLPFs requires frequency-dependent calibration for a zeroIF 802.11a RX.

\section{DISCUSSION}

The previous analysis shows that the CBPFs have better and less frequency-dependent $I M R(\omega)$. In complex filters [3$6,8]$ the image is gradually filtered while passing through it. The overall leakage of a complex filter is given by a "leaking-filtering" iterative process $[8,13]$. Therefore, the I/Q mismatch of the first stage(s) matter more than of the last stage(s). However, in case of a pair of real filters the image is "cancelled" at the global output only, since there is
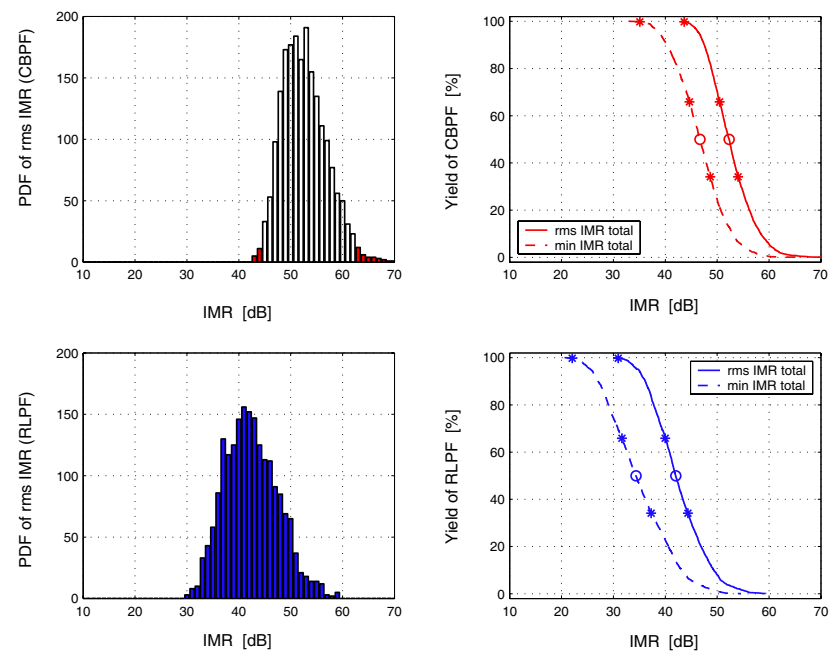

Figure 6: Histograms and yield curves for CBPF and RLPF. no interaction between the I-path and Q-path internal nodes. Therefore, the I/Q mismatch of each stage equally matters, since the image is not attenuated "internally."

Complex low-pass filters were proposed for zero-IF transceivers in [13]. The I/Q leakage mechanism in band-pass and low-pass complex filters is the same. However, bandpass complex filters better reject the image than their lowpass counterpart since they operate at a higher IF than DC. Therefore, their I/Q imbalance is lower.

\section{CONCLUSION}

In this paper a "frequency-dependent" statistical analysis was proposed which revealed that $\operatorname{IMR}(\omega)$ resembles the shape of the group delay. Also, it showed a predominantly "flat" complex bandpass filter, while the statistical $\operatorname{IMR}(\omega)$ is highly dependent on $\omega$ for a pair of real low-pass filters. As an numerical example, a system-level Monte-Carlo analysis was carried out for a 7-pole 17$\mathrm{MHz}$ bandwidth 10-MHz IF complex band-pass ladder filter prototype. It showed large image rejection ( $I M R_{\mathrm{rms}}>43.7 \mathrm{~dB}$ for $3 \sigma)$. The same filter, but reconfigured as a pair of real low-pass filters, achieved about 13-dB less rms and $\min I M R$. Therefore, the I/Q imbalance of two-path filters is significantly lower in low-IF than zero-IF architectures. In conclusion, the presented complex band-pass ladder filter eliminates the frequency-dependent I/Q calibration needs in low-IF 802.11a wireless LAN receivers.

\section{REFERENCES}

[1] B. Razavi, RF Microelectronics, Saddle River: Prentice Hall, 1998.

[2] "Part 11: Wireless LAN medium access control (MAC) and physical layer (PHY) specifications. High-speed physical layer in the $5-\mathrm{GHz}$ band," IEEE Standard 802.11a, 1999.

[3] D. Gabor, "Theory of communication," Journal of the Institution of Electrical Engineers, vol. 93, part III, pp. 429-457, 1946.

[4] M. J. Gingell, The Synthesis and Application of Polyphase Networks with Sequence Asymmetric Properties, Ph.D. thesis, Faculty of Engineering, University of London, 1975, http://users.vnet.net/gingell/.

[5] W. M. Snelgrove and A. S. Sedra, "State-space synthesis of complex analog filters," in Proceedings of the European Conference on Circuit Theory and Design, 1981, pp. 420-424.

[6] A. S. Sedra, W. M. Snelgrove, and R. H. Allen, "Complex analog bandpass filters designed by linearly shifting real low-pass prototypes," IEEE ISCAS'85, pp. III.1223-III.1226.

[7] R. H. Allen, "Complex analog filter obtained from shifted lowpass prototypes," M.S. thesis, University of Toronto, 1985.

[8] S. A. Jantzi, Quadrature Bandpass Delta-Sigma Modulation for Digital Radio, Ph.D. thesis, University of Toronto, 1997.

[9] A. I. Zverev, Handbook of Filter Synthesis, NY: Wiley \& S., 1967.

[10] R. Schaumann and M. E. van Valkenburg, Design of Analog Filters, New York: Oxford University Press, 2001.

[11] L. Yu and W. M. Snelgrove, "A novel adaptive mismatch cancellation system for quadrature IF radio receivers," IEEE Trans. on Circuits and Systems-II, vol. 46, no. 6, pp. 789-801, June 1999.

[12] G. Palaskas, Y. Tsividis, and V. Boccuzzi, "A power efficient channel selection filter / coarse AGC with no range switching transients," IEEE Custom Integrated Circuits Conference, Sept. 2003, pp. 21-24.

[13] P. Kiss, V. Prodanov, and J. Glas, "Complex low-pass filters," Analog Int. Circuits and Signal Processing, vol. 35, pp. 9-23, Apr. 2003. 